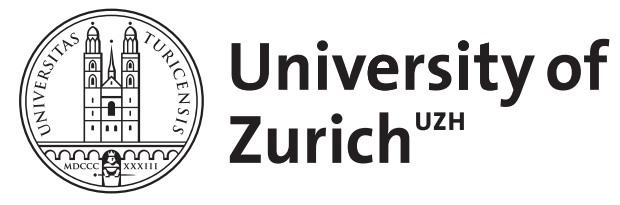

\title{
Relativism about predicates of personal taste and perspectival plurality
}

Kneer, Markus ; Vicente, Agustin ; Zeman, Dan

DOI: https://doi.org/10.1007/s10988-016-9198-z

Posted at the Zurich Open Repository and Archive, University of Zurich ZORA URL: https://doi.org/10.5167/uzh-160915

Journal Article

Published Version

Originally published at:

Kneer, Markus; Vicente, Agustin; Zeman, Dan (2017). Relativism about predicates of personal taste and perspectival plurality. Linguistics and Philosophy, 40(1):37-60.

DOI: https://doi.org/10.1007/s10988-016-9198-z 


\title{
Relativism about predicates of personal taste and perspectival plurality
}

\author{
Markus Kneer ${ }^{1} \cdot$ Agustin Vicente $^{2,4} \cdot$ Dan Zeman $^{3}$
}

Published online: 17 December 2016

(C) Springer Science+Business Media Dordrecht 2016

\begin{abstract}
In this paper we discuss a phenomenon we call perspectival plurality, which has gone largely unnoticed in the current debate between relativism and contextualism about predicates of personal taste (PPTs). According to perspectival plurality, the truth value of a sentence containing more than one PPT may depend on more than one perspective (subjects, experiencers or judges). Prima facie, the phenomenon engenders a problem for relativism and can be shaped into an argument in favor of contextualism. We explore the consequences of perspectival plurality in depth and assess several possible responses on behalf of advocates of relativism.
\end{abstract}

Keywords Predicates of personal taste $\cdot$ Relativism $\cdot$ Contextualism · Perpsectival plurality

Dan Zeman

danczeman@gmail.com; dancristian.zeman@ehu.es

Markus Kneer

markus.kneer@gmail.com

Agustin Vicente

agustin.vicente@ehu.es

1 Department of History and Philosophy of Science, University of Pittsburgh, 1017 Cathedral of Learning, 4200 Fifth Avenue, Pittsburgh, PA 15260, USA

2 Department of Linguistics and Basque Studies, University of the Basque Country, 5 Unibertsitateko Ibilibdea, 2nd Floor, Office No. 2.21, 01006 Vitoria-Gasteiz, Spain

3 Department of Linguistics and Basque Studies, University of the Basque Country, 5 Unibertsitateko Ibilibdea, 1st Floor, Office No. 1.53, 01006 Vitoria-Gasteiz, Spain

4 IKERBASQUE, Basque Foundation for Science, Maria Diaz de Haro 3, 6th floor, 48013 Bilbao, Spain 


\section{Introduction}

Predicates of personal taste (henceforth PPTs)—expressions like 'tasty', 'fun', 'disgusting', 'boring', 'cool' etc.- -have been at the center of ongoing debates in contemporary semantics. Although there is no definitive characterization of PPTs in the literature, ${ }^{1}$ one feature that most authors agree upon is their semantic dependence on subjects, experiencers or judges. To circumvent the debate regarding which of the latter three notions is most appropriate, we will take PPTs to be semantically dependent on perspectives broadly conceived. This dependence is perhaps best brought to the fore by attending to the fact that utterances of the same sentence containing a PPT can vary in truth value across contexts in which the relevant perspectives are different. To have a working example, imagine John and Marie, two eight-year olds who have just tried licorice for the first time. John found it tasty, but Marie did not. Assume that each of them separately utters

\section{(1) Licorice is tasty.}

Intuitively, the truth values of the two utterances of (1) differ: John's utterance of (1) is true, while Marie's is false. What explains this variation in truth value is the fact that John liked the taste of licorice, while Marie was repelled by it-that is, a difference in John and Marie's perspectives.

The two mainstream views that have shaped the recent debate surrounding PPTs are contextualism and relativism. Although we will focus on the latter in this paper, they are best defined in tandem. We thus assume a broadly Kaplanian framework in which sentence types or characters and contexts jointly determine the content of an utterance. Contents, in turn, are evaluated at a series of parameters drawn from the context of utterance, which form the circumstances of evaluation. Circumstances standardly comprise a time and a world, but might well include locations, epistemic standards or-our focus here-perspectives. Although contextualism and relativism capture the perspective-dependence of PPTs equally well, they do so differently. In the framework sketched above, the difference comes down to attributing distinct roles to perspectives in the semantic apparatus. According to contextualism, the perspectives on which the interpretation of PPTs depends are specified in the content of the utterances of sentences containing them. According to relativism, by contrast, the perspectives on which the interpretation of PPTs depends are values of a specific parameter in the circumstances of evaluation with respect to which the relevant utterances are evaluated. This difference can be expressed formally as follows, where (2) captures the contextualist truth conditions of (1) and (3) its relativist truth conditions:

(2) [[Licorice is tasty]] ${ }^{c, w}=1$ iff licorice is tasty-according-to- $p$ in $w$.

(3) [[Licorice is tasty]] ${ }^{c, w, p}=1$ iff licorice is tasty in $w$ according to $p$,

\footnotetext{
1 See for instance Lasersohn (2005), Stephenson (2007), Sæbø (2009), Collins (2013) and Snyder (2013) for interesting discussion.
} 
where $c$ signifies the context of utterance, $w$ the parameter for possible worlds and $p$ that for perspectives (other possible parameters are ignored for simplicity). As can be seen, in (3) there is a parameter for perspectives in the circumstances, while in (2) there is not, the perspective entering directly into the content, and as a result making it vary across contexts (the predicate 'tasty-according-to- $p$ ' is meant to capture precisely that). ${ }^{2}$

Though contextualism and relativism differ in their approaches to formalizing the perspective-dependence of PPTs, the view that PPTs do give rise to such dependence is uncontroversial. In this article, we explore a related phenomenon that has received very little attention so far, namely the possibility of perspectival plurality of sentences invoking more than one predicate of personal taste. In Sect. 2 we briefly introduce perspectival plurality. In Sects. 3 and 4, we show how plurality gives rise to two problems for relativism, one regarding the operator argument, the other regarding syntactic binding. In Sect. 5 we canvass several responses the relativist might give. In Sect. 6 we explore what might be the relativist's best shot to cope with perspectival plurality, the 'paraphrasing strategy'. Though this approach has considerable merits, it is ultimately found incapable of resolving the problems for relativism raised by plurality.

\section{Perspectival plurality}

Sentences like (1) are mono-perspectival. They contain a single PPT and thus are dependent on a single perspective in a particular context-even if said perspective is shared by a group of subjects. But consider the following examples ${ }^{3}$ :

(4) We took the kids to a resort in Italy this summer. The wine was delicious and the water slide was great fun.

(5) On Halloween, the kids did the rounds. They either got a delicious treat, or else played a silly prank on us neighbors.

(6) Even your vegetarian husband would love the new restaurant. The steaks are of course delicious, but the broccoli burgers are very tasty, too.

\footnotetext{
${ }^{2}$ Both contextualism and relativism come in many forms. Some contextualist proposals treat PPTs as containing variables for perspectives in their logical form (e.g., Stojanovic 2007; Schaffer 2011). Others conceive of PPTs as akin to gradable adjectives, and therefore as manifesting a more complex structure than a simple variable for perspectives (Glanzberg 2007). Finally, PPTs could be treated as indexicals, perhaps following Rothschild and Segal's (2009) treatment of color terms. Relativist positions differ with respect to the type of context that provides the value of the perspective parameter. So-called 'moderate' versions of relativism argue that the perspective value is drawn from the context of utterance (e.g., Kölbel 2004; Recanati 2007; Brogaard 2008). More 'radical' versions take it to be provided by the context of assessment (e.g., Lasersohn 2005, 2013; MacFarlane 2014). An orthogonal issue arises as to whose perspective is relevant in interpreting an utterance containing a PPT. Usually, the relevant perspective is taken to be that of the speaker, but this need not be so: it could be that of the salient person in a context, that of a group (as Recanati 2007; Huvenes 2012 have suggested), a generic perspective (e.g., Stojanovic 2007; Moltmann 2010; Snyder 2013), or any of the above, depending on the context (Cappelen and Hawthorne 2009). Since our discussion below is not affected by any choices made in connection to these issues, we ignore questions pertaining to them in what follows.

3 The examples and parts of the following sections are taken from or based on Kneer (2015).
} 
(7) At the world chemistry conference, professionals were encouraged to bring their partners. The talks were very interesting and the DIY experiments a lot of fun.

(8) At the educational book fair, every parent bought their child an informative yet fun new book.

Examples (4)-(8) contain multiple perspective-dependent predicates. ${ }^{4}$ Though all examples allow for various interpretations, the default reading of each relativizes the different predicates to different contextually salient perspectives. In example (4) and (5), for instance, it makes sense to assume that the adults considered the wine delicious or the Halloween pranks silly, whereas the treats were delicious for the children, just as the water-slide was fun from a point of view which is exclusively theirs, or at least includes it. Example (6) invokes different culinary tastes: a vegetarian pallet versus a non-vegetarian one. The steak will only please the latter, whereas the broccoli burger will find fancy with vegetarians, or vegetarians and non-vegetarians alike. This shows that sentences featuring predicates of taste (and others) can - and in perfectly standard cases $d o$-give rise to multi-perspectival readings, i.e., readings which draw on multiple perspectives provided by a single context of utterance. ${ }^{5}$

\section{Perspectival plurality and the operator argument}

Perspectival plurality is an interesting phenomenon in its own right. It might also constitute an important, to date largely unexplored dimension in the ongoing debate regarding which type of semantic framework-relativist or contextualist-best accommodates perspectival predicates. We will examine perspectival plurality with regards to two central arguments that have had a significant impact in the literature: the operator argument and the argument from binding. We will begin with the former.

According to a standard Kaplanian picture, we said, the truth-value of a sentence depends on the context of utterance and the circumstance of evaluation: The semantic value of a character, or sentence type, is a function from contexts and circumstances to truth-values, and the semantic values of a content, or sentence-in-context, is a function from circumstances to truth-values. But what determines which kinds of parameters the circumstance is to comprise of? 'The amount of information we require from a circumstance' Kaplan writes, 'is linked to the degree of specificity of contents, and thus to the kinds of operators in the language.' Hence, '[w] hat sorts of intensional operators to admit seems [...] largely a matter of language engineering. It is a question of which features of what we intuitively think of as possible circumstances can be sufficiently well defined and isolated.' (1989, p. 502)

\footnotetext{
4 'Informative' is not a PPT, but for all that matters here it works in the same way. What is informative for a pre-school child is not necessarily informative for an adult, and vice versa. More generally, what this shows is that perspectival plurality is not a phenomenon restricted to predicates of personal taste only.

5 In two studies in experimental linguistics (Kneer 2015, ms.) it is shown that a large majority of ordinary language speakers do interpret examples like (4)-(8) as depending on multiple perspectives. The phenomenon is also shown to extend to claims invoking multiple epistemic modal expressions.
} 
Kaplan, who conceives of operators as modifying contents of sentences, ${ }^{6}$ argues, for instance, that time must be amongst the parameters of the circumstances. His reasons are twofold: (i) tenses in English, he holds, are best conceived as sentential operators $^{7}$; and (ii) given that the purpose of temporal operators consists in shifting the time of evaluation, they must operate on entities that are variable in truth across times. Kaplan explains:

If we built the time of evaluation into the contents (thus removing time from the circumstances leaving only, say, a possible world history, and making contents specific as to time), it would make no sense to have temporal operators. To put the point another way, if what is said is thought of as incorporating reference to a specific time [...], it is otiose to ask whether what is said would have been true at another time [...]. Temporal operators applied to eternal sentences (those whose contents incorporate a specific time of evaluation) are redundant. (1989, p. 503)

Philosophers who consider claims regarding taste, aesthetics and morality to be truth-relative advocate a 'proliferation' of parameters that accommodates the relevant types of perspective. Although not necessary for relativism (cf. e.g., MacFarlane 2014), operator arguments can help motivate such parameter proliferation. Kölbel (2009), for instance, argues along these lines that the English language contains (something like) an operator that shifts the standard of taste, $\ulcorner$ FOR $t\urcorner$. If FOR is prefixed to a singular term designating an individual, the taste claim $p$ in a construction $\ulcorner$ FOR $\mathrm{t}, \mathrm{p}\urcorner$ must be evaluated with regards to $t$ 's taste. 'For Anna, whale meat is tasty', for instance, is true if whale meat agrees with Anna's pallet, i.e., if 'Whale meat is tasty' is true for Anna, or from her perspective. The taste operator is characterized by the following rules:

(S1) For all sentences $\varphi$ and all singular terms $\alpha,\ulcorner$ FOR $\alpha, \varphi\urcorner$ is a sentence.

(S2) For all $\varphi, \alpha, w, s$ and $a$ : if $\varphi$ is a sentence and $\alpha$ is a personal name referring to $a, w$ is a possible world, and $s$ is a [perspective]: $\ulcorner$ FOR $\alpha, \varphi\urcorner$ is true in a circumstance $\langle w, s\rangle$ iff $\varphi$ is true in $\langle w, \mathrm{~s}(a)\rangle$ (where $\mathrm{s}(a)$ is $a$ 's [perspective]) (2009, p. 384)

Does our ordinary language term 'for' work in ways equivalent to the taste operator FOR? Kölbel argues it does. Just as one can make a case for the existence of modal operators by aid of examples such as (9), one can adapt them to make a similar case for the existence of taste operators in ordinary English. (10), it seems, is no less true in certain contexts than its modal pendant, (9):

(9) In possible world $W$, whales are extinct, but whales are not extinct.

(10) For Anna, whale meat is tasty, but whale meat is not tasty. (2009, p. 10)

\footnotetext{
6 This premise is questioned by various scholars, cf. for instance Lewis (1980) and Richard (1981).

7 King (2003), amongst others, famously disagrees.
} 
What is more, in combination with the quantifying expressions 'everyone' and 'someone' the FOR operator mirrors the standard modal operators 'necessarily' and 'possibly'. 'FOR everyone, $p$ ' and 'FOR someone, $p$ ' shift the taste parameter in just the same ways 'necessarily' and 'possibly' shift the world parameter. ${ }^{8}$

Though prima facie not implausible, this argument in support of a taste or perspective parameter, and the taste-neutral contents it entails, runs into trouble when confronted with multi-perspectival readings. As Cappelen and Hawthorne (2009, p. 75 , fn. 10) point out, sentences like (11), at least on some interpretations, invoke more than a single perspective:

(11) Maria ate something that was tasty for Anna in a dignified way.

On the most salient reading, 'tasty' is relativized to Anna's perspective, yet 'dignified' is not-it is, much rather, dependent on what the speaker deems dignified. But if not all perspective-dependent predicates fall under the scope of the perspective operator FOR, then the latter cannot be a sentential operator in the vein proposed by Kaplan.

'Dignified', one might object, is not a predicate of personal taste, so it is no surprise that it does not fall under the 'standard of taste' operator which manipulates 'tasty'. The circumstance might include various perspective parameters, one related to personal taste, another one to style. Naturally, even if one restricts oneself to predicates pertaining to taste proper, it doesn't take much to fix the example, as Kölbel—who provided example (13)—is ready to admit:

(12) Frank showed John how to cook something tasty for his wife in a fun way.

(13) She ate something tasty for Anna on a pretty plate. (2011, p. 144)

In (12) the perspective relevant for the interpretation of 'tasty' is that of John's wife (as specified by the phrase 'for his wife'), while the one relevant for the interpretation of 'fun' is (presumably) John's. Similarly, in (13) the perspective relevant for the interpretation of 'tasty' is Anna's (as specified by the phrase 'for Anna'), while the one relevant for the interpretation of 'pretty' is (presumably) the speaker's. Assuming that the two for-phrases are modeled after Kölbel's FOR operator, since FOR is a sentential operator, we get the result that the perspectives relevant for the interpretation of the whole sentences (and thus of both PPTs) in (12) and (13) are those of John's wife and Anna's, respectively. Since this follows from the semantics of FOR, the multi-perspectival readings of (12) and (13) are excluded - a bad result.

We should be clear that we discuss this objection to the operator argument with the sole purpose of illustrating how perspectival plurality has been appealed to in the debate we are concerned with, and that we don't necessarily commit to its dialectical effectiveness. Thus, it might be possible for Kölbel to avoid the objection engendered by plurality. In fact, in responding to Cappelen and Hawthorne's

\footnotetext{
${ }^{8}$ The possibility of FOR combining with variables is taken by Kölbel to follow straightforwardly from the definition of the operator. See Kölbel (2011, p. 145) for discussion.
} 
counterexample, he suggests to conceive of 'for' as an intensional predicate operator rather than a sentential operator-a view that is shared by Lasersohn (2008) and MacFarlane (2012). According to such a view, 'for Anna' operates on a single PPT ('tasty') and not on all PPTs that occur in the sentence. While such an approach is reasonable in itself, it is no longer clear whether it has much argumentative bite, since restricting the scope of such operators from sentences to predicates means that we have little in the way of an operator argument along Kaplanian lines. ${ }^{9}$ Another way out for Kölbel would be to claim that the relevant for-phrases only sometimes work as sentential operators, which would be enough for the introduction of a parameter for perspectives in the circumstances. ${ }^{10}$ Finally, a last line of defense would be to cling to the initial assumption that they are fully sentential operators and recast the problematic examples in ways that preserve it (see, e.g., Lihoreau 2012). Discussing such answers in detail would take us too far afield; suffice it to say that perspectival plurality blocks sentential operator arguments of the type discussed under standard interpretations of its premises.

\section{Perspectival plurality and the binding argument}

The second argument in the context of which perspectival plurality occurs is the discussion of the Binding Argument. Lasersohn (2008) provides a response to this argument, which has been recently used against relativism about PPTs (e.g., Schaffer 2011). ${ }^{11}$ In a nutshell, the argument starts from the premise that there are bound readings of the target expression, and via certain bridging principles between syntax and semantics concludes that the target expression has a variable of a certain kind in its syntactic configuration. Since claiming that an expression has a variable of a certain kind in its syntactic configuration (whose value enters into the content of utterances of sentences containing the expressions in question) amounts to a contextualist view about that expression, the argument supports such a view over the rival relativist view. Schaffer constructs the argument against relativism about PPTs on the basis of the bound reading of

\section{(14) Everyone got something tasty}

where the thing that each person under the range of 'everyone' got is tasty for that person - that is, the perspective to which 'tasty' pertains to is bound. Schaffer concludes that 'tasty' has a variable for perspectives in its syntactic configuration.

\footnotetext{
9 See, however, Zeman (2013) for a reconstruction of the operator argument without taking the relevant phrases to be sentential operators.

${ }^{10}$ We thank an anonymous reviewer for suggesting this alternative.

11 The argument from binding has been employed for a variety of expressions by Stanley in a number of papers now collected in Stanley (2007). Stanley, however, was concerned with unarticulated constituents, that is, propositional constituents not articulated at the surface level of the sentence. These, he suggested, should not be conceived as pragmatically supplied variables, but as implicit arguments, which are part of the syntax of the expression.
} 
Lasersohn avoids this conclusion by providing an alternative account of binding that allows one to 'quantify directly on the [perspective parameter], setting and resetting its value in tandem with the variable introduced by a quantifier' (2008, p. 324). This alternative account_-index-binding'—replaces quantification over variables for perspectives in the object language with quantification over such variables in the meta-language-in the present case, over the parameter for perspectives in the circumstances of evaluation ('index', in Lasersohn's jargon). Formally, this idea is implemented by introducing a second sentence-abstractforming operator, $\mu$, in addition to the more common $\lambda$-operator, and to treat quantifier phrases as such sentence-abstract forming operators. This treatment allows quantifier phrases to bind both variables in the object language and variables in the meta-language (parameters in the index) when the variable in the metalanguage is of a different kind than the one in the object language. Thus, when they bind variables in the object language, their effect could be described as in (15); when they bind variables in the meta-language-and here we focus specifically on the parameter for perspectives - their effect could be described as in (16):

$$
\begin{aligned}
& {[[\lambda n \varphi]]^{M, c, w, u, g}=\left\{x \in U \mid[[\varphi]]^{M, c, w, u, g[x / n]}=1\right\} \quad(2008, \text { p. 313) }} \\
& {[[\mu n \varphi]]^{M, c, w, u, g}=\left\{x \in U \mid[[\varphi]]^{M, c, w, x, g[x / n]}=1\right\} \quad(2008, \text { p. 324), }}
\end{aligned}
$$

where $\varphi$ is a sentence, $M$ is a model, $c$ a context, $w$ a possible world, $u$ an individual, ${ }^{12} g$ an assignment function, $g[x / n]$ is that sequence in which $x$ is the $n$-th element and which agrees with $g$ in all other positions and $U$ the set of individuals. As (15) shows, $\lambda$ manipulates the assignment function (the familiar lambdaabstraction), whereas, as (16) shows, $\mu$ manipulates the parameter for the perspective in the index, so that the truth of $\varphi$ is dependent on the values taken by $x$. To illustrate how this complicated account works, let us consider the following example:

(17) Every man rode some ride that is fun.

(17) is represented in Lasersohn's system as

(18) $\left[[\right.$ every man $] \mu_{1}\left[\left[\right.\right.$ some [ride that $\lambda_{2}\left[\mathrm{pre}_{2}\right.$ is-fun $\left.\left.]\right]\right] \lambda_{3}\left[\operatorname{pre}_{1}\left[\operatorname{rode}\right.\right.$ pre $\left.\left.\left.\left._{3}\right]\right]\right]\right]$,

where the quantifier phrase 'every man' binds both a variable for individuals in the object language $\left(p r \theta_{1}\right)$ and the parameter for perspectives in the index. Thus, Lasersohn claims, '[(17)] receives a reading in which each man rode a ride which is fun for him, even though there is no variable corresponding to for him in the syntactic representation. The index itself is 'bound', but this is part of our metalanguage, not the object language.' (2008, p. 325).

\footnotetext{
12 We interpret Lasersohn's parameter for individuals $u$ as a parameter for perspectives. Since perspectives are held by (at least) one individual, the modification is innocuous.
} 
All its technical ingenuity notwithstanding, index binding has considerably less expressive power than variable binding, as Lasersohn is ready to admit. Once there are multiple PPT in a phrase, the variable approach allows for multiple binding, since each PPT might come equipped with its own syntactic variable. In index binding, by contrast, the quantifier phrase binds the single perspective parameter. Lasersohn's example, (19), could in principle be interpreted in (at least) four different ways $(19 \mathrm{a}-\mathrm{d})$ :

(19) Every man gave some woman a fun ride and a tasty dish. (2008, p. 325)

(19a) Every man gave some woman a ride and a dish, which were tasty and fun according to the speaker.

(19b) Every man gave some woman a ride and a dish, which were tasty and fun according to each man.

(19c) Each woman received a ride and a dish, which were tasty and fun according to her standards.

(19d) Every man gave some woman a ride fun by his standards, as well as a dish tasty according to her standards.

Index binding can represent readings (19a)-(19c) without problems, because each of these interpretations invokes only a single perspective. This, Lasersohn writes, makes perfect sense of one basic tenet of relativism:

[In index binding] we are employing a single, systematic parameter, relative to which all denotations are assigned; and if an operator manipulates this parameter, it will do so for all expressions in its scope. (...)

The intuition behind this pattern can perhaps be expressed this way: In a relativist theory, in order to assess a sentence for truth or falsity, one must adopt a stance-that is, truth assessment is always done from a particular perspective. Operators in the sentence may shift the perspective from which truth assessment is to be done, or quantify over such perspectives; and when they do so, the relevant perspective must be adopted for the entire scope of the operator. Because such operators shift the perspective from which truth is assessed, rather than shifting the denotation of some particular expression like a pronoun, they cannot selectively shift only certain items in their scope. (2008, p. 326)

So far, so good. However, in order to make sense of (19d)-the interpretation in which the two PPTs are relativized to two different perspectives-we need flexible variable binding. The hidden argument of 'fun', on a contextualist rendition of this interpretation, is bound by 'every man', whereas the variable of 'tasty' is bound by 'some woman'. According to Lasersohn, this is not a problem, because 'the sentence [i.e., (19)] cannot mean that each man gave some woman a ride that was fun for him, and a dish that was tasty for her' (2008, p. 325, italics added). This, in turn, he takes to 'show that [PPTs] cannot have arguments freely chosen from a set of pronouns similar to $\operatorname{pro}_{1}$, pro $_{2}$, pro $_{3}, \ldots$ ' (2008, p. 326). Now, we agree with Lasersohn that the interpretation (19d) of his example sentence (19) may sound 
rather awkward, and that it is not easy to get a multi-perspectival reading of that sentence. ${ }^{13}$ However, this does not mean that claims involving multiple PPTs never allow for perspectival plurality. In fact, in all of the examples (4)-(8) provided above, the multi-perspectival reading is not only perfectly felicitous, but arguably constitutes the default interpretation. Naturally, similar examples can be devised to include quantifiers, and thereby make a case in favor of multiple perspective variables in a single claim:

(20) On Halloween, every child would play a silly trick on some adult or else get a delicious treat.

(21) Every Argentinean took some vegetarian friend to the Sunday barbecue for some tasty steaks and delicious corncobs.

(22) On father's day, the fair was in town. Every dad took some child to the fairground to taste the delicious local brew and try out the fun new rides.

(23) Every historian took an engineering friend along to the university fair and there were great new robots and highly interesting history talks.

One perfectly felicitous interpretation of (20), for instance, would have it that the victims of the Halloween pranks, i.e., the adults, consider them silly, whereas the recipients of the treats, i.e., the kids, consider said treats delicious. This is not only a perfectly plausible reading, but the default reading: the kids won't find their own tricks silly, whereas the adults quite possibly think little of the cheap chocolate handed out to appease the youngsters. To take just one more example, on the most natural interpretation of (22), the perspective variable of the 'delicious local brew' is bound by 'every dad', whereas the variable of the 'fun ride' is bound by 'some child'. Lasersohn's discussion of (19) shows that, by means of index binding, the relativist can accommodate a considerable number of interpretations of claims involving multiple PPTs-namely, all the ones in which the various PPTs are relativized to a single perspective. However, it is clear that the limited expressive power of index binding is insufficient to account for all the readings of sentences similar to (19) — in particular, for the multi-perspectival readings illustrated by our examples (20)-(23).

Though our principal focus lies with relativism, a brief note on contextualism. Prima facie, plurality can be easily accommodated by contextualism, given that on such an approach each PPT comes part and parcel with its own free perspective variable. There is no restriction regarding what type of perspective can saturate the variable, even in cases of coordination as the above. Variables can be filled by an exocentric perspective, by a generic perspective or the subjective perspective of the speaker. In cases where a sentence contains multiple PPTs, there is no reason to suppose that the two different variables need to be saturated by the same perspective or even the same type of perspective. Hence, contextualism does not face the same obstacles generated by plurality as relativism does.

\footnotetext{
13 The reading might be acceptable given enough contextual support. However, instead of trying to devise a context where the multi-perspectival reading of (19) sounds fine, we will make use of more natural (but structurally equivalent) examples.
} 


\section{Relativist responses}

\subsection{Available options}

We have suggested that perspectival plurality engenders two important problems for relativism: It questions the possibility of justifying the postulation of perspective parameters by aid of operator arguments, and it raises severe difficulties to account for binding. We see two types of responses that can be made on behalf of the relativist: A negative strategy would attempt to challenge the central premises of our arguments. A positive strategy would amend the relativist framework so as to accommodate plurality.

As regards negative options: To deal with both arguments wholesale, what must be undermined is the empirical premise common to both, that is, the premise that ordinary language speakers do in fact interpret the above example sentences in multi-perspectival fashion. However, in a series of five studies in experimental semantics involving over 600 participants, Kneer (ms.) finds conclusive proof for perspectival plurality both with respect to taste claims and epistemic modals. Given that a wholesale attack on the empirical plausibility of plurality is thus unlikely to succeed, relativists will have to question the conceptual assumptions of each argument in a piecemeal fashion.

Though we here assume that parameters must be justified, and that one of the most plausible ways to do so consists in operator arguments à la Kaplan, alternative strategies can be envisioned. ${ }^{14}$ Furthermore, the requirement to account for syntactic binding could be called into question, for instance on the basis that embedding is not semantically innocent ${ }^{15}$ or that the binding argument overgenerates. ${ }^{16}$ Pursuing such questions in depth would carry us too far afield. We would merely like to note that this line of defense is only successful if both the binding argument and the requirement for parameter justification are brought to fall, most likely on independent grounds since they make different assumptions. Note that the view that parameter proliferation must be justified by an operator-type argument and consistent with syntactic binding is a common one and shared by many relativists. Undermining the premises of both arguments would have considerable revisionary consequences, which might be seen as both an ad hoc and excessive response to the phenomenon of perspectival plurality.

Alternatively, a positive proposal could be envisaged, which amends the relativist framework in ways suitable to accommodate perspectival plurality. We can think of two ways this could, at least in principle, be achieved: further parameter proliferation or 'multi-indexing' on the one hand, or else a general strategy according to which complex sentences invoking multiple PPTs are broken down into mono-perspectival units whose truth is evaluated individually. In the following

\footnotetext{
14 MacFarlane (2009, p. 245), for instance, casts doubt on Stanley's (2005, pp. 69-72) contention that parameter proliferation can only be motivated through operator arguments.

15 Cf. Recanati (2002), cf. also Sennet (2008) for helpful discussion.

16 Cf. for instance Capellen and Lepore (2002, p. 274) and Recanati (2002); for a response cf. Martí (2006).
} 
we will briefly voice some reservations as regards the first option (Sect. 5.2). The second, novel, alternative - the 'paraphrasing strategy' — will be developed in a bit more depth in Sect. 6, as we consider it the relativist's best shot. We ultimately find fault with this type of response, too, and must conclude that the problems plurality raises for relativism are not easily overcome.

\subsection{The uniqueness of perspective constraint}

In Sects. 3 and 4 we have seen that perspectival plurality poses a challenge for relativism both with respect to operator arguments and with respect to syntactic binding. As regards the latter, one might think that the problem lies in a certain particularity of Lasersohn's account of binding. But, as the passage we quoted from Lasersohn makes clear, the problem runs deeper: according to Lasersohn himself, it is a core commitment of relativism that is in tension with the multi-perspectival readings we have put forward. Lasersohn's reason for rejecting such readings of (19) had to do with the adoption of a stance, which amounts to the claim that each sentence has to be evaluated at a unique perspective. ${ }^{17}$ Let us flag this 'one sentence -one stance-one perspective' claim for further reference, by dubbing it the

\section{Uniqueness of Perspective Constraint (UPC)}

A sentence has to be evaluated for truth relative to one and only one perspective of a certain kind.

We take it that the UPC follows from a natural view of what relativist semantics is. ${ }^{18}$ Relativists claim that perspectives belong to circumstances of evaluation in the Kaplanian divide between content and circumstances of evaluation, along with world and time parameters. These parameters are functions from contents to truthvalues. This explains why there can only be one of each of these parameters per content evaluated: the work of each parameter is to help determine whether a certain content is true or false (or true or false relative to the other parameters). What doesn't seem feasible is to have two perspective parameters (or two world or time parameters) in the circumstances of evaluation. It would be feasible to have two different perspective parameters if these worked at the local level, i.e., at the level of the predicate, before the content is built by a process of composition. However, if perspectives work at the global level, i.e., the level of sentential content, there seems to be no way to make two different parameters work in the way the relativist would

\footnotetext{
17 A more detailed discussion about taking a stance in the relativist framework can be found in Lasersohn (2009, Sect. 3).

18 Kissine (2012) makes the same claim about what he calls 'circumstance-of-evaluation-relativism'which is what we simply call 'relativism'. Although his focus is a relativist view about gradable adjectives, the objection he raises involves multi-perspectival readings in which one of the perspectives is made explicit. His objection, however, is more complex in the sense that he investigates short exchanges in which one of the interlocutors use the locution 'I agree' to signal the fact that there is agreement concerning a conjunctive sentence whose conjuncts each contains a gradable adjective that is to be evaluated with respect to a different comparison class. One premise in his argument against relativism about gradable adjectives is the assumption that each conjunctive sentence has to be evaluated with respect to a unique comparison class-that is, he assumes the UPC.
} 
want them to work. In other words, the UPC is in tension with perspectival plurality, and the problem is general. ${ }^{19}$

Before we move on, a clarificatory remark concerning the precise formulation of the UPC above. According to the constraint, a sentence has to be evaluated for truth relative to one and only one perspective of a certain kind. As various philosophers have pointed out, relativists face a choice as regards the determinate set-up of the circumstances. Each type of perspective-dependent expression (aesthetic predicates, taste predicates, epistemic modals etc.) might require its own parameter. Or else, one might combine them all into a 'catch-all standard of evaluation' (Kölbel 2009, p. 384), i.e., a general perspective parameter which all (or most) types of such expressions depend on. The latter approach is just as problematic as the first, if not even more so, in particular since it means that sentences invoking multiple perspectives are even more ready at hand. Consider:

(24) The cat food might be tasty. (Stephenson 2007, p. 499, credited to Danny Fox)

Stephenson employs (24) to argue that PPT can, whereas epistemic modals cannot, be understood relative to an exocentric perspective. This alleged difference is, in our view, rather spurious, but off-topic. What the example can be used to show, however, is that a catch-all parameter for perspectival features is problematic: There simply seems no plausible alternative to a reading according to which the tastiness of the cat food must depend on the cat's perspective, and the epistemic modal 'might' on the speaker's perspective. ${ }^{20}$ What this suggests is that advocates of parameter proliferation best help themselves to different parameters for different sorts of perspectives, so as to avoid that the problems here raised arise in even more drastic form with regards to a single catch-all parameter. However, some of the

\footnotetext{
19 The problem posed by perspectival plurality can be circumvented if one thinks that the semantics of PPTs doesn't need to be uniform. Thus, MacFarlane (2014) thinks that PPTs that are used differently get different treatments: if a PPT is used exocentrically (that is, from another's perspective) or is bound, the predicate has a variable for perspectives in its logical configuration, while if a PPT is used egocentrically (that is, from one's own perspective) the variable is lacking. (A similar idea appears in Stephenson (2007), where both uses of PPTs have a variable for perspectives in their logical configuration, but the variables are of different types (pro and $\mathrm{PRO}_{\mathrm{J}}$ ).) Since perspectival plurality consists in various combinations of egocentric, exocentric and bound uses of PPTs, this approach avoids the problem because there is always one perspective the sentence will be evaluated against-that relevant for the interpretation of the predicate(s) used egocentrically-thus rescuing the UPC. However, MacFarlane doesn't provide any justification for the different treatment of the egocentric, exocentric and bound uses of PPTs, and a solution of this sort thus sounds rather ad hoc. Besides being ad-hoc, this approach makes PPTs ambiguous between a one-place predicate (when used egocentrically) and a two-place predicate (when used exocentrically and bound), thus leading to a hybrid (that is, relativist and contextualist) view. Ambiguity, we take it, is better avoided as a semantic solution and used only as a last resort.

${ }^{20}$ Kölbel (2009) mentions another problem in passing. Having introduced a FOR operator for taste predicates (see above) and a POSSIBLY operator for epistemic modality he acknowledges that 'the two operators might cause some complication to do with scope. Consider, for example, the sentence 'Whale meat might have been tasty for Anna'. Should this be construed as 'POSSIBLY (FOR Anna, whale meat is tasty)' or as 'FOR Anna, POSSIBLY whale meat is tasty'?' Given that we already run into problems regarding scope when different parameters depend on the outlook of a single individual, things are bound to get considerably worse if different parameters invoke different perspectives.
} 
examples above show that it is simply not possible to save relativism by distinguishing different kinds of perspectives. It can be said, concerning our example (5), for instance, that whereas 'tasty' is clearly a predicate of personal taste, 'silly' is a different kind of predicate, which relates to a different kind of perspective. This move is not feasible in cases like (6), where we find two PPTs proper, 'delicious' and 'tasty'. We doubt that we can individuate kinds of perspectives in a way so fine-grained that we could get relativism off the hook in this way. ${ }^{21}$

\subsection{Multiple perspective parameters}

The train of thought proposed in the previous section, perhaps, moves a little too quickly. Relativism, one might hold, is either committed to the UPC, or must provide a plausible picture according to which there are multiple perspective parameters with regards to a particular domain. But contrary to what was alleged above, there is good precedent regarding multiple parameters of the same sort, at least as concerns time. In fact, multi-indexing was subject to extensive debate in the 1970s, and if the strategy holds promise regarding time, it might carry over to various sorts of perspective parameters. Kamp (1971) and Vlach (1973) argued that expressions such as 'now' and 'then' call for double temporal indexing in frameworks that treat tense as index-shifting sentence operators. Consider the following examples:

(25) One day, all persons alive now will be dead.

(26) Once all persons alive then would be dead.

The sentence in (25) makes reference to two different times, the time of utterance, as well as a certain day in the future when everyone alive at the time of utterance is dead. The truth conditions of (25) cannot appropriately be expressed by invoking a standard Priorean future tense operator only. The time the indexical expressions 'now' or 'at the present moment' refer to would also be shifted into the future by the future tense operator. What is required (it seemed) is double temporal indexing, that is, one index shiftable by tense operators plus another rigid one which gets picked up by the indexical 'now'. The first index corresponds to the time of evaluation, the second to the time of reference.

Though we will not investigate the possibility of imitating this strategy with respect to perspective parameters in detail, two brief considerations why it holds little promise. Firstly, whether multi-temporal indexing is a helpful idea is highly contentious. King (2003, pp. 222-223) for instance, demonstrates that there are good syntactic reasons to treat tenses as quantifiers over times rather than as index shifting sentence operators. But if the temporal index shifting operators are best

\footnotetext{
${ }^{21}$ We thank an anonymous reviewer for pressing this point. Let us add that the problem related to perspectival plurality does not arise only with respect to PPTs. It is also possible to devise examples where two different epistemic modals (e.g., two different occurrences of 'might') in a sentence relate to two different perspectives (Kneer 2015, ms.). In these cases, it makes no sense to argue that the two occurrences of 'might' relate to different kinds of perspectives.
} 
avoided then, King concludes, we don't need temporal coordinates either. So the hope to mimic multi-index approaches in tense logic as regards other parameters might prove frustrating: syntactic concerns pertaining to multiple time coordinates bring out the attractiveness of time-specific (or eternalist) propositions. Multiplying other parameters to account for perspectival plurality might raise similar worries which, ultimately, make a case for taste-specific propositions and the like. Naturally, the attempt to spell out such an argument makes little sense in the absence of a detailed story - to be provided by the relativist-regarding how such a multiple perspective parameter picture is to work in the first place.

Secondly and more importantly, even in the absence of a detailed proposal, it seems that the analogy between multiple time indexing and the multiple taste indexing we would require to circumvent the problems posed by our examples is a poor one. In the tense-logic case, multiple indexing is motivated by the fact that certain indexicals like 'now' (as well as expressions which incorporate some indexical element like 'the current president of the US') leap out of the scope of the tense operator. The problem posed by 'now' is resolved (if it is) by postulating two very different time coordinates, each of which has a specific function. The time of evaluation interacts with tense and is shiftable, the time of reference interacts with temporal indexicals and is unshiftable.

In our examples above, however, such a clear-cut division of labor is not available: different occurrences of PPT must be evaluated relative to the same type of thing - a personal standard of taste - and hence the same type of parameter or coordinate. So whereas the different temporal parameters discussed are devised in such a way that they can only interact with the relevant parts of the sentence, multiple taste parameters in our examples are, as it were, 'unoriented'. The problem is that none of the PPTs in our examples so far behaves like a reference-fixing device (we will discuss examples in which PPTs behave like this below). These examples involve PPTs that are of equal status: either both of them contribute with a perspective to the content of the sentence, or both of them relate to perspectives in the circumstances of evaluation. The relativist is committed to this latter view, but such a view is in conflict with the UPC.

\section{The paraphrasing strategy}

The relativist, we said, faces a dilemma: holding fast to the UPC means plurality is left unaccounted for, accommodating the latter means giving up a central tenet of relativism. A final attempt to disarm the dilemma consists in modifying the UPC in ways that reduce the scope of Lasersohn's 'one stance' commitment from entire sentences to meaningful units of truth-evaluation. Differently put, Perspectival uniformity could be satisfied at the level of individual sub-sentences, while the truth of the content as a whole might still be relative to multiple perspectives. Consider this revised version of the UPC:

\section{Uniqueness of Perspective Constraint* (UPC*)}

A unit of evaluation has to be assessed for truth relative to one and only one perspective of a certain kind. 
Restricting the UPC in scope makes room for the following proposal: Suppose that multi-perspectival sentences can be paraphrased as concatenations of simpler propositional units, each of which contains but one single PPT. The resultant monoperspectival units can be evaluated individually in conformity with the UPC* (i.e., invoking not more than one perspective each). The truth-value of the sentence as a whole is calculated on the basis of the values of the individual units. Although the truth-value of each individual unit is relative to a single perspective at most, the truth-value of the content of the sentence as a whole can be sensitive to multiple perspectives.

\subsection{Applications and fundamental premises}

Very roughly, the paraphrasing strategy, as we will call it, assumes that multiperspectival claims can be paraphrased into a series of mono-perspectival units joined by logical connectives. Furthermore, the strategy requires that the process of truth-assessment can be conceived (or at least reconstructed) as proceeding in a piecemeal fashion in which the truth-value of the sentences as a whole is determined on the basis of the values of its individual units. To see how the strategy might deal with some of the problematic cases, let's begin with a simple example. A complex sentence such as (27) is factored out into a conjunction of two simpler individual units, (28):

(27) Mary has a biscuit and a coffee.

(28) Mary has a biscuit \& Mary has a coffee.

Assessing (27), the suggestion goes, involves assessing 'Mary has a biscuit' and 'Mary has a coffee' individually. Once a verdict for each of the two individual units has been reached, the latter are used to compute the truth-value of the complete content in conformity with the basic rules of propositional calculus. (27) is true if and only if both of the conjuncts of (28) are true. Things are no different when it comes to sentences containing PPT. (29), for instance, can be paraphrased as (30):

(29) Mary had a fun ride and ate a tasty dish.

(30) Mary had a ride \& Mary ate a dish \& the ride was fun \& the dish was tasty.

Just as in the previous example, (29) is true if and only if every single one of the conjuncts of (30) is true. In line with the UPC*, the individual units 'the ride was fun' and 'the dish was tasty' must be evaluated with respect to exactly one perspective each. Since they are assessed individually, however, nothing prevents us from using different perspectives for each of the units. Whether this is advisable depends, of course, on which perspectives are salient in the particular context at hand. For instance, in a context in which the speaker considers himself an authority in culinary matters, but defers to experiencers as regards rollercoasters, the ride might be evaluated as fun relative to Mary's perspective, yet the dish as tasty relative to the speaker's perspective. The UPC* is satisfied with respect to each of 
the conjuncts containing a PPT, while the truth-value of the sentence as a whole can still be sensitive to more than one perspective.

Consider further (31), which does not contain a conjunction but a conjunctive DP. (31) can be paraphrased as (32):

(31) Mary had a fun ride and a tasty dish.

(32) Mary had a ride \& Mary had a dish \& the ride was fun $\&$ the dish was tasty.

Again, the perspective parameters of the two individual units containing PPTs can be set to different values, and again (31) is true iff all of the conjuncts of (32) are true-potentially with respect to distinct perspectives.

So far we have outlined a very general strategy relativists might pursue in order to counter the worries posed by perspectival plurality. Fleshing out this proposal in a satisfying manner would constitute a paper in itself. Here we limit ourselves to briefly sketching some of the core assumptions on which the paraphrasing strategy relies. There are at least four such assumptions:

A1: Sentences that contain $n$ PPTs can always be decomposed into at least $n$ units of evaluation.

A2: Each unit of evaluation will contain no more than one PPT in a predicative position. $^{22}$

A3: The truth of each constitutive unit can be assessed independently.

A4: The truth of the entire sentence can be derived from the truth values of its individual units.

Each of the assumptions raises problems and would saddle the relativist with commitments that go well beyond the problematic here raised. We will briefly discuss a few of these.

\subsection{Assumption A1: the possibility of paraphrasing}

Though A1 merely states that multi-perspectival claims can be factored out into multiple units which involve at most one perspective, a satisfying account must state a general recipe for the paraphrasing process. One possibility to do so focuses on atomic sources of error. Suppose we want to assign a truth-value to the sentence 'This is a green tomato'. We would check whether the designated object is a tomato, and thereafter whether the object is green. Given that there are two potential sources of error, i.e., two ways in which the sentence can misrepresent the world, one needs to have an eye on each. Since there are two sources of error that must be assessed independently, the sentence must be broken down into two distinct units of evaluation. Each unit is assessed individually, and the value 'true' can be assigned to the sentence as a whole if both sources of error can be ruled out, that is, if each unit is evaluated as true. This strategy is rather general in nature, because it does not

\footnotetext{
22 The reason we talk here about predicative position will become clear in Sect. 6.3.
} 
engender further commitments with respect to whether 'green' and 'tomato' denote properties, sets, or functions, although it is in principle plausible that the way we verify whether a predicate applies to a subject is by thinking about the predicate as denoting a property.

Alternative theories of truth-value assignment can be envisioned. Here is one: when evaluating 'This is a green tomato' we focus on the intersection of the set of green things and the set of tomatoes (or the conjunctive property green tomato) and check whether the demonstrated object is present in the set (or whether the object instantiates the conjunctive property). On this proposal, there would be just one possible source of error and so just one unit of evaluation. When evaluating sentences with multiple PPTs in predicative position, we have to construe the intersection of several sets or properties, each of which may relate to a different perspective. Suppose we say

(33) Marie had a funny-looking, tasty dish,

where the dish was funny-looking to us and tasty for Marie. If we use this type of verification procedure, then before we assign a truth-value to (33) we have to contextualize the denotations of 'funny-looking', and of 'tasty', so that the truth or falsity of (33) would not be relative to a perspective. Naturally, this type of verification procedure is at odds with the paraphrasing strategy and the trouble plurality spells for relativism is back full force. What this means is that adopting the paraphrasing strategy engenders a commitment to the first type of verification procedure, which tracks atomic sources of error. The best support for this view would be evidence that demonstrates that ordinary language speakers in fact proceed along these lines in truth-value assessment. Unfortunately, very little is known about the psychology of truth-assignment to sentences in natural language, ${ }^{23}$ so a claim of this would constitute a somewhat daring empirical conjecture. Alternatively, one might consider it sufficient to provide a plausible, rational reconstruction of said underlying psychological process, which would be subject to revision once empirical linguistics makes progress. Proposals of this sort are not uncommon. For example, on some interpretations Grice's theory of implicature should be understood as a plausible rational reconstruction of the psychological process that underpins the processing of implicatures. ${ }^{24}$ Advocates of the paraphrasing strategy would hence need to provide some general, empirically plausible rationale

\footnotetext{
23 See, however, Pietroski et al. (2009) for some partial results regarding the expression 'most'. The authors compare different ways ('verification procedures') of assigning truth-values to 'most' sentences. Such verification procedures yield truth-conditions that are extensionally equivalent, but they are very different in computational terms. They focus in particular on the 'one-to-one correspondence strategy' and the 'cardinality comparison strategy' as applied to a simple sentence such as 'most $x$ s are F' (e.g., 'most dots are blue'). The one-to-one correspondence strategy in a simple setting where only Fs and Gs are relevant, consists in checking (i) whether for each thing that is an F there is a corresponding thing that is a $\mathrm{G}$, and then (ii) whether there is an excess of Fs, thus verifying that most $x$ s are F. The cardinality comparison strategy compares the cardinalities of the Fs and the Gs and assigns truth if the cardinality of the Fs is bigger than the cardinality of the Gs. After running some experiments, their conclusion is that we assign a truth-value to a 'most' sentence by comparing cardinalities.
}

24 Cf. for instance Geurts and Rubio-Fernández (2015). 
regarding why truth-value computation might proceed in piecemeal fashion in the first place and, on the basis of the former, a recipe of how the complex sentence is to be broken down into smaller, meaningful units.

\subsection{Assumptions A2 and A3: mono-perspectival units and independent truth-assessment}

Let's turn to A2, according to which each unit of evaluation must contain no more than one PPT in a predicative position. As an anonymous reviewer suggested, certain types of adverbs might give rise to tricky cases. Consider:

(34) The dog food is astonishingly tasty.

Since the dog food itself cannot reasonably be deemed astonishing, it is not obvious how the two PPTs ${ }^{25}$ in predicative position can be pulled apart so as to form two distinct units of evaluation. Perhaps (34) could be factored out into (35):

(35) The dog food is tasty \& this is astonishing.

Some support that a paraphrase along these lines is plausible comes from authors like Katz (2005) and Nouwen (2005) who have argued that 'astonishingly', in sentences such as the one under consideration here, behaves like a special intensifier or degree modifier such as 'very', not as a sentential modifier as is sometimes assumed. Hence, (34) entails 'the degree to which the dog food is tasty is astonishing'. ${ }^{26}$ Although this seems a viable rendering of (35), the relativist is saddled with certain assumptions about 'astonishing' that might not be completely innocuous.

Note, on the other hand, that paraphrasing (34) as (35) raises a worry regarding the independence of the conjuncts (A3 above): the use of the demonstrative 'this' in the second conjunct of (35) explicitly refer back to the entire first conjunct. Naturally, it seems unreasonable to ban any connection between conjuncts whatsoever - in 'John played a silly prank and he had a lot of tasty licorice', 'he' refers back to John yet paraphrasing it as two conjuncts doesn't look problematic. However, the more interdependence is deemed acceptable, the more assumption A3 comes under pressure, that is, the assumption that the truth of the distinct units can

\footnotetext{
${ }^{25}$ Strictly speaking, 'astonishing' is not a PPT. However, it is similar enough as it is also a perspectivedependent expression in the relevant sense.

26 This way to go might amount to a satisfactory solution due to the fact that, after all, it is possible to claim that we can distinguish two truth-evaluable units in (35). A first unit is expressed by the claim: 'the dog food is tasty'. This unit has to be evaluated according to a perspective (the dog's perspective) and to the standard fixed by the context. Once it is decided whether the dog food is tasty for the dog according to the standard we have fixed (a standard related to the price we paid, for instance), we have to decide whether it is astonishing to find the dog's food in the point of the scale of tastiness where we find it. In order to do this, we do not have to relativize again to the dog's tastes. The dog's tastes were relevant to fixing what counts as tasty, and thus to evaluating the first atom. Now, in the second step, we are only concerned with whether finding the food there, where we find it, is astonishing.
} 
be assessed independently from one another. While we will not pursue this matter further here, suffice it to say that the relativist needs to clearly specify the sense in which the two units are taken to be independent from each other, and ensure that A3 holds good.

Assumption A2 seems to come under pressure from other expressions as well. Consider:

(36) The book was fun yet informative. ${ }^{27}$

Paraphrasing (36) into mono-perspectival units such as 'The book was fun' \& 'the book was informative' loses the contrastive force of 'yet' and it is not clear what should be added in order to account for it. Perhaps one could follow Potts (2005), according to whom the contrastive force of 'yet' can be captured in a separate unit such as 'For the most part, interesting books are not fun'. But the problem merely resurfaces, since an extra unit of this sort contains (37), which itself can be multiperspectival:

(37) Interesting books are not fun.

Here is a possible solution. As mentioned above (assumption A2), the defender of the paraphrasing strategy is committed to the idea that we will not find more than one PPT in a predicative position in each of the units of evaluation corresponding to any given sentence. As we hope it is clear, (37) has a multi-perspectival reading according to which the books are interesting for the parents but not fun for the kids. Nonetheless, it seems that in (37) there is a single unit of evaluation and two PPTs. Now, it could be said that in order to know whether (37) is true we have to first find the interesting books (i.e., books that are interesting for us, parents), and then check whether those books are fun, i.e., whether the kids find them fun. That is, the PPT in the subject position, 'interesting', helps us determine the extension of the NP. Once we have that extension, we only have to check whether it is true that the individuals in the set have the property of being fun. To evaluate this we have to relativize to the kids' perspective. In sum, once we have fixed the denotation of the NP, the unit of evaluation contains just one PPT: 'the books in the set (the ones parents find interesting) are not fun'. One of the PPTs would contribute to the content of the sentence, while the perspective related to the other one, the one in the VP, which has a predicative use, is located in the circumstances of evaluation. This move would draw on the multi-indexing strategy explained above. The reason why multiindexing would be allowed in this case (if it is) is that the different PPTs play different roles in the sentence: one has a referential role, while the other is purely predicative.

In the previous section we contended that it was not possible to account for our examples (4)-(8) and (20)-(23) by distinguishing two roles perspectives might play:

\footnotetext{
27 If one worries about 'informative' not being a PPT, here is an example with two PPTs in the strict sense: 'I took the kids to Pomp, Duck \& Circumstance. The dinner was fun yet delicious.'
} 
supplying information to the content, and being part of the circumstances of evaluation. In a sentence like 'the current president of the US is handsome', we can distinguish two elements sensitive to time. Each plays a different role: the first ('current') has a role to play in building the content by fixing a reference, while the second (the verbal tense) relates to an index in the circumstances of evaluation. (At least, that would be the relativist's story.) Now, as all our examples until then concerned two PPTs that were in straightforward predicative positions, both of them had to play the same role: either both contributed to the content (as the contextualist would have it) or both related to different indexes in the circumstances of evaluation (which could be a relativist move, but we said we do not see how it can be implemented). However, example (37) is different. Example (37) is structurally analogous to the example involving 'the current president...', so the relativist seems to be allowed to deal with it in a similar way, i.e., by treating the first PPT as contributing to fixing the reference of the object of predication.

Now, even if responses such as the ones tried here were to be seen as satisfactory, we are convinced that there are many more examples that will pose problems for a paraphrasing strategy (particularly ones involving quantification, as no food is both tasty and amusing). While we take it that the strategy is probably the best option for the relativist, we leave the question whether it is indeed a promising strategy open.

\subsection{Assumption A4: truth-value computation}

The final assumption, A4, requires that it be possible to derive the truth-value of the complex sentence from the truth-values of the individual units. This premise, too, can be called into doubt. One problem regards the question whether the constituent units into which the complex sentence gets split up provide sufficient guidance in deciding relative to which perspectives they must be evaluated. It is standard practice to speak about context 'providing' or 'supplying' the relevant parameters of the circumstance of evaluation (Kaplan 1989, p. 591). This manner of speaking, however, considerably oversimplifies what is actually going on. In evaluating a sentence in context, it is frequently not the case that context puts a ready-formed world-time-perspective tuple at the interlocutor's fingertips. Instead of the passive reception of the 'provided' parameters, at times an active investigation of the context is required, in particular when the options are plentiful (as, for example, in the wine and water-slide example, which allows for at least twelve reasonable different readings as regards perspective).

Lewis, for instance, highlights that some features of context are more accessible than others. Whereas certain features such as time, place, world and the speaker might be relatively obvious, ' $[\mathrm{t}]$ he audience, the standards of precision, the salience relations, the presuppositions (...) of the context are given less directly. They are determined, so far as they are determined at all, by such things as the previous course of the conversation that is still going on at the context, the states of mind of the participants, and the conspicuous aspects of their surroundings.' (1980, p. 86). The question thus arises whether paraphrasing, giving rise to a (slight) change in cotext, and thus context, will leave the salience of perspectives unchanged. Differently put, relative to which perspective the first constitutive sentence must be evaluated, 
and relative to which the second, might either be less obvious, or once torn apart, the PPTs in the individual constituent sentences might call for different perspectives than the ones that prove salient when the sentence is evaluated as a whole.

Another, not unrelated worry: The precise mechanics of truth-evaluation according to the paraphrasing strategy might be deemed somewhat mysterious. The constitutive sentences of a multi-perspectival claim get evaluated separately, the proposal goes, each with regards to a single, appropriate perspective. Since parameters are 'provided', 'supplied' or 'given' by context, and since the perspective parameters for each of the simple sentences differ, do we not require two different contexts, too? If we understand contexts, with Lewis, as concrete situations of utterance, it is important to recall that 'features of context do not vary independently. No two contexts differ by only one feature. Shift one feature only, and the result of the shift is not a context at all.' (1980, p. 86; cf. also Kaplan's ban on improper contexts, though recall that for Kaplan 'contexts' are technical constructs.) Now, if there are several units of evaluation instead of one complex one, there may also be several contexts (differing in terms of more than just one feature), and thus several circumstances of evaluation or indices. These indices might in principle differ considerably, since they are determined by different contexts. So assumption 4, too, is far from innocent. All this is not to say that the paraphrasing strategy cannot be eventually made to work, or that the relativist is out of options in general. What it does show, however, is that perspectival plurality constitutes a rather serious challenge to relativism, and that overcoming it is not a simple affair.

\section{Conclusion}

In this paper we have introduced a phenomenon we called 'perspectival plurality', according to which the truth value of a sentence with multiple PPTs can depend on more than one perspective. Plurality, we suggested, might raise difficulties for those who attempt to justify parameter proliferation in the circumstance of evaluation by means of Kaplanian operator arguments. What is more, the phenomenon blocks what might be considered the most promising relativist account of binding.

We assessed two broad relativist strategies to respond to plurality. We rejected the first-denying the data-by pointing to robust experimental results obtained by Kneer (ms.). The second broad strategy we considered accommodates plurality at the conceptual level. Two proposals of this sort were explored: the postulation of multiple perspective parameters, and what we called the 'paraphrasing strategy'. Whereas we raised doubts that the former is viable at all, the latter, we argued, holds at least some initial promise. However, certain tricky sentences might resists paraphrasing, and a number of more general worries may afflict this approach, too. Further research will prove whether the strategy can be successfully developed, or whether perspective plurality turns out to be an insuperable obstacle to relativism.

Acknowledgements Markus Kneer would like to thank Peter Pagin, Max Kölbel, Isidora Stojanovic, François Recanati, John Perry, Edouard Machery and Robyn Carston, and the audience of the 2014 PLM 
workshop in San Sebastián for helpful feedback on a dissertation chapter on which parts of this article are based. Agustin Vicente and Dan Zeman's research for this work has been funded by Projects IT769-13, from the Basque Government, and FFI2014-52196-P, from the Spanish Ministry of Economy and Competitiveness (MINECO). Dan Zeman also acknowledges the financial help of Project FFI201237658, as well as that of a Juan de la Cierva grant, both funded by MINECO. Both authors would like to thank Elena Castroviejo and Javier Ormazabal, the other members of the HiTT group at the University of the Basque Country and the audiences at the 2015 PLM workshop in Oslo, the 1st Context, Cognition and Communication conference in Warsaw and the Pervasive Context conference in Reading. All three authors thank the two anonymous referees for this journal for their suggestions and comments.

\section{References}

Brogaard, B. (2008). Moral contextualism and moral relativism. The Philosophical Quarterly, 58, 385409.

Cappelen, H., \& Hawthorne, J. (2009). Relativism and monadic truth. Oxford: Oxford University Press. Cappelen, H., \& Lepore, E. (2002). Indexicality, binding and a priori truth. Analysis, 62, 271-281.

Collins, J. (2013). The syntax of taste. Philosophical Perspectives, 27, 51-103.

Geurts, B., \& Rubio-Fernández, P. (2015). Pragmatics and processing. Ratio, 28, 446-469.

Glanzberg, M. (2007). Context, content, and relativism. Philosophical Studies, 136, 1-29.

Huvenes, T. T. (2012). Varieties of disagreement and predicates of taste. Australasian Journal of Philosophy, 90, 167-181.

Kamp, H. (1971). Formal properties of 'now'. Theoria, 37, 227-274.

Kaplan, D. (1989). Demonstratives. In J. Almog, J. Perry, \& H. Wettstein (Eds.), Themes from Kaplan (pp. 481-563). Oxford: Oxford University Press.

Katz, G. (2005). Attitudes toward degrees. In E. Maier, C. Bary, \& J. Huitink (Eds.), Proceedings of SuB 9 (pp. 183-196). Nijmegen: Radboud Universiteit Nijmegen.

King, J. (2003). Tense, modality and semantic values. Philosophical Perspectives, 17, 195-245.

Kissine, M. (2012). From contexts to circumstances of evaluation: Is the trade-off always innocuous? Synthese, 184, 199-216.

Kneer, M. (2015). Perspective in language. Dissertation, Institut Jean Nicod/ENS Paris.

Kneer, M. (ms.). Perspectival plurality in natural language.

Kölbel, M. (2004). Indexical relativism vs genuine relativism. International Journal of Philosophical Studies, 12, 297-313.

Kölbel, M. (2009). The evidence for relativism. Synthese, 166, 375-394.

Kölbel, M. (2011). Objectivity, relativism and context dependence. Hagen: Fern Universität Hagen.

Lasersohn, P. (2005). Context dependence, disagreement, and predicates of personal taste. Linguistics and Philosophy, 28, 643-686.

Lasersohn, P. (2008). Quantification and perspective in relativist semantics. Philosophical Perspectives, 22, 305-337.

Lasersohn, P. (2009). Relative truth, speaker commitment, and control of implicit arguments. Synthese, 166, 359-374.

Lasersohn, P. (2013). Non-world indices and assessment-sensitivity. Inquiry, 56, 122-148.

Lewis, D. (1980). Index, context, and content. In S. Kanger \& S. Ohman (Eds.), Philosophy and grammar (pp. 79-100). Dordrecht: Reidel.

Lihoreau, F. (2012). Personal taste ascriptions and the Sententiality assumption. The Reasoner, 6, 143144.

MacFarlane, J. (2009). Nonindexical contextualism. Synthese, 166, 231-250.

MacFarlane, J. (2012). Relativism. In D. Graff Fara \& G. Russell (Eds.), The Routledge companion to the philosophy of language (pp. 132-142). New York: Routledge.

MacFarlane, J. (2014). Assessment sensitivity: Relative truth and its applications. Oxford: Oxford University Press.

Martí, L. (2006). Unarticulated constituents revisited. Linguistics and Philosophy, 29, 135-166.

Moltmann, F. (2010). Relative truth and the first person. Philosophical Studies, 150, 187-220.

Nouwen, R. (2005). Monotone amazement. In P. Dekker \& M. Franke (Eds.), Proceedings of the fifteenth Amsterdam Colloquium (pp. 167-172). Amsterdam: ILLC. 
Pietroski, P., Lidz, J., Hunter, T., \& Halberda, J. (2009). The meaning of 'most': Semantics, numerosity and psychology. Mind and Language, 24, 554-585.

Potts, C. (2005). The logic of conventional implicatures. Oxford: Oxford University Press.

Recanati, F. (2002). Unarticulated constituents. Linguistics and Philosophy, 25, 299-345.

Recanati, F. (2007). Perspectival thought: A plea for moderate relativism. Oxford: Oxford University Press.

Richard, M. (1981). Temporalism and eternalism. Philosophical Studies, 39, 1-13.

Rothschild, D., \& Segal, G. (2009). Indexical predicates. Mind and Language, 24, 467-493.

Sæbø, K. J. (2009). Judgment ascriptions. Linguistics and Philosophy, 34, 327-352.

Schaffer, J. (2011). Perspective in taste predicates and epistemic modals. In A. Egan \& B. Weatherson (Eds.), Epistemic modality (pp. 179-226). Oxford: Oxford University Press.

Sennet, A. (2008). The binding argument and pragmatic enrichment, or, why philosophers care even more than weathermen about 'raining'. Philosophy Compass, 3, 135-157.

Snyder, E. (2013). Binding, genericity and predicates of personal taste. Inquiry, 56, 278-306.

Stanley, J. (2005). Knowledge and practical interests. Oxford: Oxford University Press.

Stanley, J. (2007). Language in context. Selected essays. Oxford: Oxford University Press.

Stephenson, T. (2007). Judge dependence, epistemic modals, and predicates of personal taste. Linguistics and Philosophy, 30, 487-525.

Stojanovic, I. (2007). Talking about taste: Disagreement, implicit arguments, and relative truth. Linguistics and Philosophy, 30, 691-706.

Vlach, F. (1973). 'Now' and 'then': A formal study in the logic of tense anaphora. Dissertation, University of California, Los Angeles.

Zeman, D. (2013). Experiencer phrases, predicates of personal taste and relativism: On Cappelen and Hawthorne's critique of the operator argument. Croatian Journal of Philosophy XIII, 39, 375-398. 